\title{
Heat transfer aspects of condensation during vapour phase soldering on oriented PCB-based surfaces
}

\author{
Dániel Straubinger ${ }^{1}$, István Bozsóki ${ }^{1}$, David Busek ${ }^{2}$, Olivér Krammer ${ }^{1}$, Balázs \\ Illés ${ }^{1}$, Attila Géczy ${ }^{1,2}$ \\ ${ }^{1}$ Dept. of Electronics Technology, Budapest University of Technology and Economics \\ Budapest, Hungary \\ ${ }^{2}$ Czech Technical University in Prague, Faculty of Electrical Engineering, \\ Department of Electrotechnology, Technickâ 2, Prague 6, 166 27, Czech Republic
}

\section{Abstract}

- Purpose: The paper presents an investigation on heat transfer in vapour phase soldering oven, focusing on the differences of horizontally and vertically aligned PCB surfaces. The investigation can lead to a better understanding of the process and information for future modelling of the process.

- Design/methodology/approach: For the investigations, FR4 PCB plates and sealed platebased boxes were immersed into the saturated vapour of an experimental oven. The temperature and resulting heat transfer coefficients were analysed according to the sample boxes and the orientations of the surfaces. In addition, the vapour consuming of the bodies was also investigated with pressure measurements.

- Findings: It was found that the horizontal top and bottom side heating results in very similar results. Also, the sides of a box were heated in a similar manner to the top and the bottom, but there is a slight increase in the heat transfer coefficient, due to the vertical wall alignment. The pressure measurements reveal the dynamic change in the vapour after immersion.

- Practical implications: The findings may help to show differences on different surface orientations, pointing to more precise explicit and multiphysics simulation results. 
- Originality/value: The experiments present an aspect of heat transfer coefficient differences in vapour phase soldering ovens, also highlighting the effect of initial pressure drop inside the workspace of an oven.

Keywords: Vapour Phase Soldering, Condensation Soldering, Reflow Soldering, Heat Transfer Coefficient

\section{Introduction}

Vapour Phase Soldering (VPS), or as it is sometimes referenced, condensation reflow soldering, is a complex heat transfer process used for reflow soldering in order to enable high-quality solder joints, achieved by even heating. Due to this aspect, the method is not only used for common Surface Mount Technology (SMT), but for assemblies with mixed component types as well, where larger power electronic components (Synkiewicz et al., 2017) are designed and assembled onto the same assembly as smaller SMT components. The standard VPS process can be described with the following steps: the assembly is lowered into the saturated vapour blanket, which is produced by Galden type PFPE fluid, this vapour is then condensed onto the colder surface. Consequently, latent heat is released due to the phase change of the vapour, this latent heat gives the energy required to melt the solder and heat up the assembly (Zabel, 2006). Soldering in VPS ovens is difficult to describe with simple modelling approaches because complex descriptions are describing the layers of the process. Such effect is the state and flow of the condensate (Illés, 2013), the heating power, volume and shape of the saturated vapour blanket and surface dimensions of the assembly.

As a possible direction of optimization, VPS can be used with vacuum conditions; it was reported, that exceptional quality can be achieved in vaccuum oven configurations (Huang et al., 2018; Dziurdzia et al., 2018; Lüngen et al., 2015). VPS ovens can be further improved by adding heating gradient control (heat level based method), more specific profile setup with soft vapour configuration (Leicht and Thumm, 2008) or another intricate sample movement in the work zone of the oven. Nowadays VPS is applied more and more frequently in mass production as well, as suggested by distributors and oven producers (Manncorp, 2019).

The most recent research on VPS focused on different application aspects. The reflow method was applied for brass solderability for hybrid sewing in smart textiles (Pavec. et al. 2019). The wettability of different solders was also recently investigated in VPS by (Molhanec, 2019). Sensor measurement fundaments of the work zone were re-evaluated by Livovsky and Pietrikova (2017). According to the basics of reflow, an optimal soldering profile and the heating factor have a considerable effect on the 
solder joint quality during VPS (Veselý et al, 2018). New sensors and measurement approaches can also be used for better control of the process (Livovsky and Pietrikova, 2019).

It is apparent from the literature, that only a few are investigating the heat transfer itself and the layered processes in depth. One of the first summary to give a proper description via an explicit description was presented by (Leider, 2002), however, a more recent approach involved a more sophisticated approach on modelling of the heat transfer on a PCB assembly in vapour (Straubinger, 2019). The description of the heat transfer is usually originated to Newton's law of heating and the relevant heat transfer coefficient derived from the wall-condensation model of Nusselt, and the consequential works of Bejan (1991).

As Thumm showed, the heat transfer coefficient for convection based reflow ovens can be found in the range of $40-60 \mathrm{~W} / \mathrm{m}^{2} \mathrm{~K}$, the same for VPS is starting from around $100 \mathrm{~W} / \mathrm{m}^{2} \mathrm{~K}$ (Thumm, 2010). Other reports start from 250 and tend to $700 \mathrm{~W} / \mathrm{m}^{2} \mathrm{~K}$ depending on the heat transfer fluid and the horizontal-vertical alignment of the samples (Leider, 2002). This value needs further investigation, while the heat transfer coefficient depends on the oven, the state of the blanket, the geometry and alignment of the assembly, and the used materials in the structure of the assembly and inside the oven.

Bejan described the heat transfer coefficient for condensation in case of a horizontal plate, and the vertical slab, with horizontal top and vertical wall surfaces (Bejan, 1991). While the first description could fit the model of a horizontal PCB (considered as a thin, horizontal wall), the components need attention from this specific aspect. The approach, where Bejan's extended model (Bejan, 1991) was used to describe the heat transfer on the components, was found to be non-universally applicable for SMD components (Straubinger, 2019). As it was found, the classical analytical models are starting to deviate from measured data, as the geometries re scaled down, and the models are extended in verticality - so a refined understanding is required from the aspect of condensation regarding all wall orientations. Further analysis is needed for three-dimensional bodies immersed in saturated vapour, to further improve the description of condensation heating during VPS. Figure 1 shows an example, where the component, the PCB, and the given geometrical sides are represented, where condensation occurs.

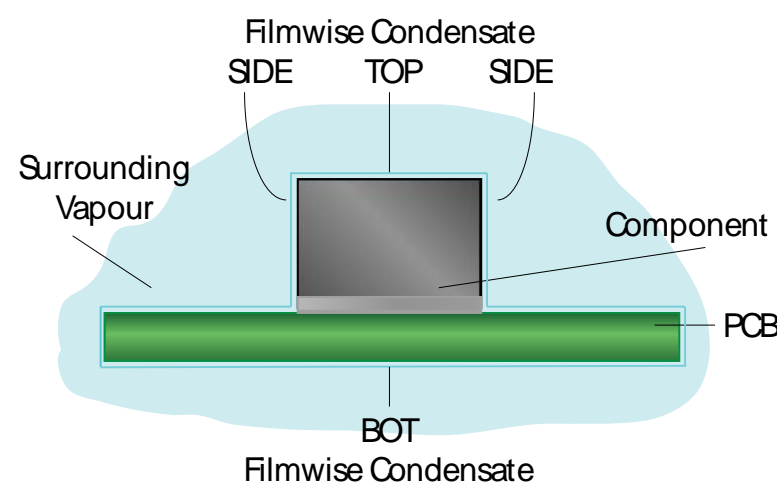


Fig. 1 Condensation during VPS.

To improve knowledge on heat transfer at different surfaces, the paper tackles a focused aspect of the topic, where the measured and calculated the heat transfer coefficient is presented between different orientations of the printed circuit board-based samples, such as FR4 plates and boxes. The results may help in the future modelling of the component, board and assembly-level heat transfer, ultimately pointing to a better understanding of the process, and thus, better quality and reliability of the produced joints.

\section{Materials and methods}

\subsection{Experimental}

For the measurements, a saturated vapour space was set in an experimental soldering station, where the used Galden was HT170 type. The heating power was set to maintain the saturated vapour space, in spite of the introduced thermal masses of the measurement samples, so it is suggested that vapour is saturated during the process.

In order to achieve a recording of the heat transfer coefficient, plates and closed boxes were made from FR4 (PCB substrate) boards. Air was trapped inside the box for thermal insulation due to practicality. The boxes were composed of different scaling aspects.

Figure 2 presents a $6 \times 6 \times 2 \mathrm{~cm}$ sized measurement box ("Box A") constructed from $1 \mathrm{~mm}$ thick FR4 boards, where one side is probed with K-type thermocouples, in the middle and in the corner. The Figure shows not only the fixing of the thermocouples inside and the leading out of the wires but the thermocouple fixing as well. The box was sealed with silicone rubber adhesive, withstanding the temperatures of the HT170 heat transfer medium. Water immersion tests were used to verify hermetic sealing in a pool of water. Next to Box A, a $6 \times 6 \mathrm{~cm}$ sized FR4 bare laminate control plate was introduced and prepared with the same thermocouple arrangement, to provide control results.

Figure 3 presents a 1x1x1 cm sized second box ("Box B") where five sides are probed with K-type thermocouples, the sixth side is used as a thermocouple leadout. The given structure is important to be able to obtain proper knowledge of separation between the different sides (horizontal/vertical) of the heat transfer.

For temperature recording, K-type thermocouples were used $\left( \pm 1{ }^{\circ} \mathrm{C}\right)$, which were fixed with Loctite 3621 type SMD adhesives into bores of the board samples. The thermocouple data were logged with a multi-channel data acquisition system, then the data was processed on PC. In the VPS oven, a proprietary (0-1 PSI) gage sensor-based pressure measurement device was also used to investigate the hydrostatic pressure drop of the vapour blanket (Alaya, 2019). 

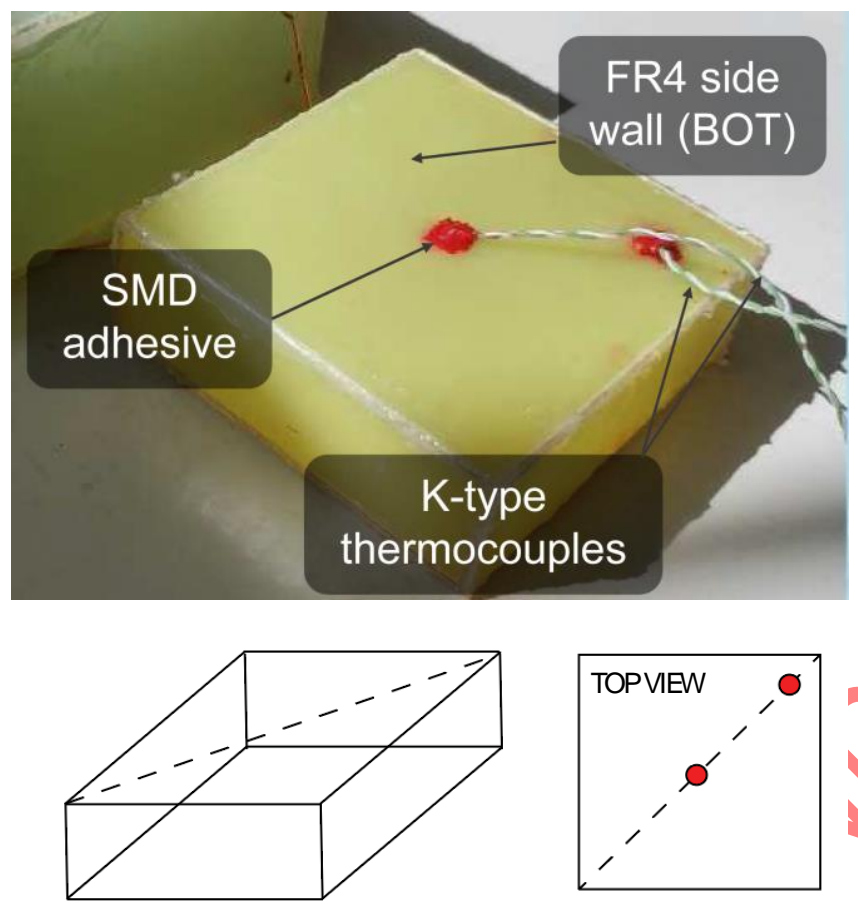

SIDEVIEW OFTHESTRUCTURE

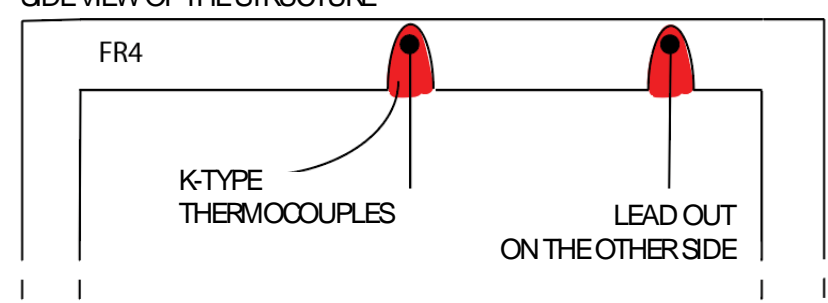

Fig. 2 (Top) Measurement "Box A", and (Bot) thermocouple attachment inside the box

Figure 3 also shows a hot gas blower heat gun validation measurement, which was used to ensure the cross-effect of different sides. The heat gun was positioned $1 \mathrm{~cm}$ from the surface of one side, then the thermocouple readings were analysed to see, if the heating of one side will cause cross-effect heating by thermal conduction to the others (Neighbour sides 1 and 2; consequently the near neighbour (1) and the further neighbour (2) sides). Note that the system was set up on the ambient laboratory environment, so the thermal differences from the heated side to the cool ones would present a worst-case, considering the $\Delta \mathrm{T}$ across the geometry. The validation measurement was set to two temperature steps on the heat gun: $140^{\circ} \mathrm{C}$ and $220^{\circ} \mathrm{C}$ (two temperatures relevant from the melting point of SnBi and SAC solder alloys). Any drop in the sensor log means, that the vapour is consumed by the immersed thermal mass of the body, slightly altering the boundary condition of saturated vapour. 


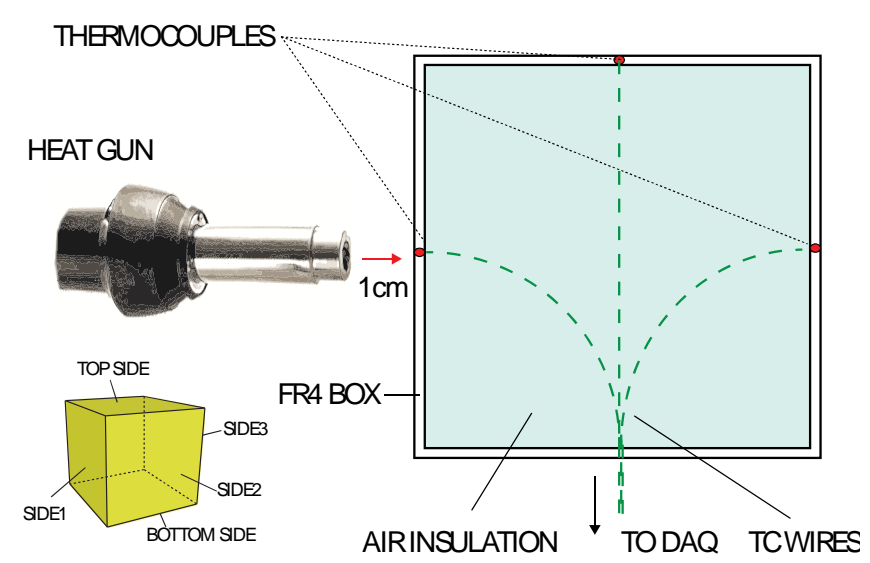

Fig. 3 (Left) "Box B" with side definition and (right) validation measurement setup

During the actual measurements in vapour, Box A and B are immersed into the saturated vapour of an experimental vapour phase oven where the top and the bottom side is always positioned to the same height inside the oven during immersion. A total of three runs were recorded for each positioning to investigate repeatability. The control plate for Box A was also immersed to the same height, where the previous side measurements were performed with the box.

\subsection{Calculation basics}

The thin FR4 laminate during VPS heating behaves like a concentrated thermal mass, with nearly equal temperature distribution across its thickness. Thus, with uniform heating, the dynamic behaviour of the laminate temperature is approximated as a first-order system (1), also known as the lumped model (Cengel and Ghajar, 2015):

$\mathrm{c} \cdot \mathrm{m} \cdot \frac{\mathrm{dT}}{\mathrm{dt}}=\mathrm{h} \cdot \mathrm{A} \cdot\left(\mathrm{T}_{\mathrm{sat}}-\mathrm{T}\right)$

where $\mathrm{c}\left[\mathrm{J} / \mathrm{kg}{ }^{\circ} \mathrm{C}\right]$ is the specific heat capacity, $\mathrm{m}[\mathrm{kg}]$ is the mass, $\mathrm{t}[\mathrm{s}]$ is the time, $\mathrm{h}\left[\mathrm{W} /\left(\mathrm{m}^{2}{ }^{\circ} \mathrm{C}\right)\right]$ is the heat transfer coefficient, $\mathrm{A}\left[\mathrm{m}^{2}\right]$ is the heated surface, $\mathrm{T}\left[{ }^{\circ} \mathrm{C}\right]$ is the temperature of the body and $\mathrm{T}_{\text {sat }}$ is the temperature of the saturated vapour.

The lumped model can be solved numerically with the time-stepping finite difference method, or under certain circumstances, an analytic solution can also be fitted to the measured data. The timestepping scheme for the heat transfer coefficient is given by:

$$
\mathrm{h}_{\mathrm{i}}=\frac{\mathrm{T}_{\mathrm{i}+1}-\mathrm{T}_{\mathrm{i}}}{\Delta \mathrm{t}} \cdot \frac{\mathrm{c} \cdot \mathrm{m}}{\mathrm{A} \cdot\left(\mathrm{T}_{\mathrm{sat}}-\mathrm{T}_{\mathrm{i}}\right)}
$$


where $i$ is the index for the actual step. The numerical time derivation of the temperature can result in unstable-noisy calculated heat transfer values. This can be the result of an inadequate resolution of the analogue-digital conversion process.

To obtain a proper heat transfer coefficient for the measurements in a second step, we applied the analytic method. we assumed, that a quick immersion into the saturated vapour results in the step response of the heated system. When every parameter is time invariant, except the laminate temperature, the analytic solution of the step response of Eq. (1) for $t \geq 0$ is:

$\mathrm{T}(\mathrm{t})=\mathrm{T}_{\text {init }}+\left(\mathrm{T}_{\text {sat }}-\mathrm{T}_{\text {init }}\right) \cdot\left(1-\exp \left(-\frac{\mathrm{t} \cdot \mathrm{h} \cdot \mathrm{A}}{\mathrm{c} \cdot \mathrm{m}}\right)\right)$

where $T_{\text {init }}$ is the initial temperature of the body.

It was found, that Eq. (3) fitted quite well in most cases, which allow us to determine the heat transfer value from the measured temperature data. The curve fitting was carried out using the weighted least squares method. The heat transfer coefficient values obtained by this way can be assumed as an average value during the condensation process.

It must be noted, that for a heated plate in vapour, the energy is obtained from both surfaces, while in the case of the box, we assume that the inner side is considered to be adiabatic, while the air does not heat up considerably during the process. This is shown in Figure 4. It is assumed, that there is no heat transfer occurring from the inner side.
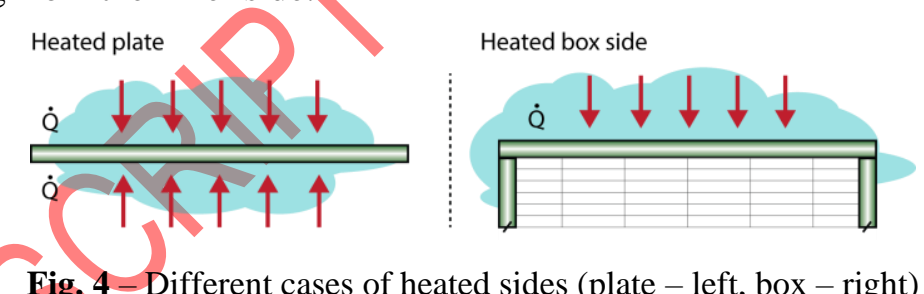

Fig. 4 -Different cases of heated sides (plate - left, box - right)

\section{Results}

\subsection{Initial evaluation of the box models}

As Figure 5 shows, the evaluation measurements give a clear representation of the 140 and $220^{\circ} \mathrm{C}$ steps caused by the hot gas blower positioned $1 \mathrm{~cm}$ from the surface of the box. Also, it is apparent, that the neighbour sides are heating in a similar fashion, despite their distance from the heated point. At the approximate $140{ }^{\circ} \mathrm{C}\left(\Delta \mathrm{T} 1_{\text {box }}\right), 14{ }^{\circ} \mathrm{C}$ increase $\left(\Delta \mathrm{T}_{\text {lneighbour }}\right)$ was recorded which is a $12 \%$ cross effect at the worst case for heat conduction (cold sides on both neighbour sides). For the next step, which gives an additional $70^{\circ} \mathrm{C}$ to the system $\left(\Delta \mathrm{T}_{2 \mathrm{box}}\right)$, an additional $12{ }^{\circ} \mathrm{C}$ increase $\left(\Delta \mathrm{T}_{2 \text { neighbour }}\right)$ was recorded at the sides, which is a $17 \%$ cross effect at the worst case for heat conduction (cold sides on both neighbour sides). It is supposed, that if all sides are heated at the same time, higher overall 
temperatures mean smaller temperature gradients across the box and the air insulation, meaning that the gradient-based heat conduction will be smaller. This means that the model box is able to record the side temperatures with negligible cross-effect errors. It is also suggested, that the air inside of the box was heated in a negligible manner for the time period of the investigations presented in later subsections.

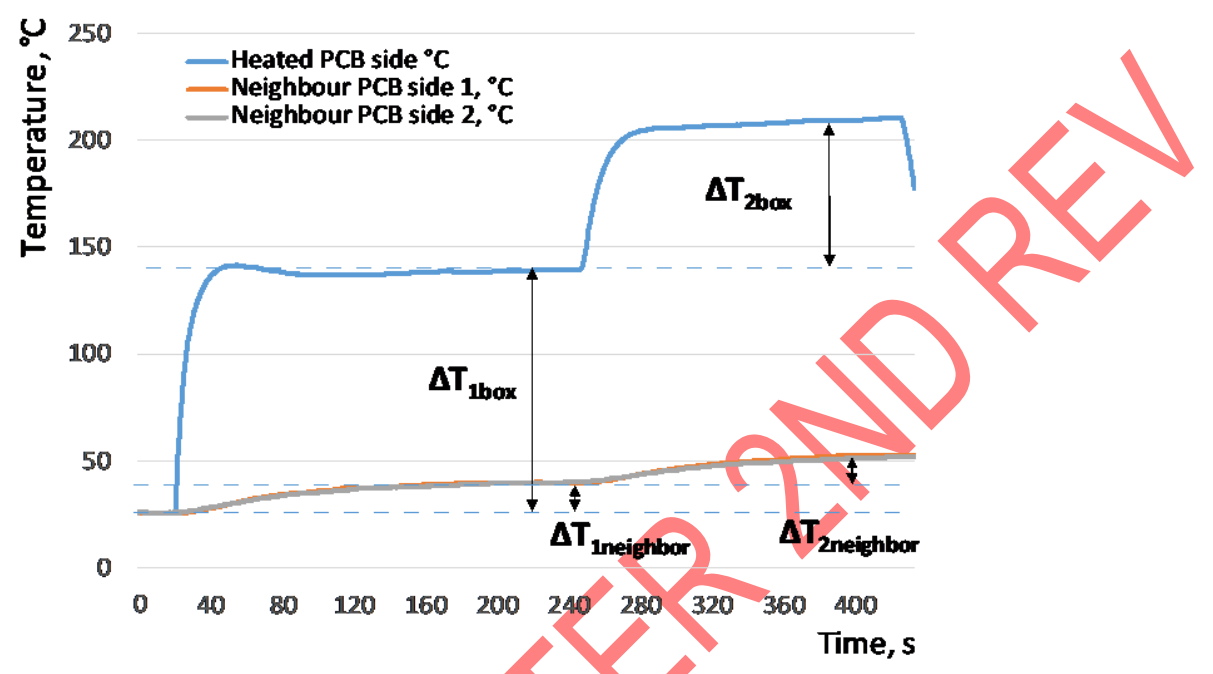

Fig. 5 Results of evaluation measurements - two step responses at two different temperatures $\left(140^{\circ} \mathrm{C}\right.$ and $\left.220^{\circ} \mathrm{C}\right)$

\subsection{Heat transfer coefficient investigations with Box A}

The plot presented in Figure 6 shows the calculated (finite difference method) and averaged heat transfer coefficient from the recorded temperatures of Box A, which provide an approximation of very similar results from both cases, where the investigated side is considered to be on either the TOP or on the BOT level. The top side has a slightly larger average value for the heat transfer coefficient, however, the difference might be negligible which is an important aspect from further modelling. The noise can be traced back to data acquisition and the sensitivity of heat transfer coefficient calculations from recorded temperatures. As the recorded data starts to tend to the maximum temperature during the exponential heating (and the resulting temperature steps will be smaller), the noise increases. It is apparent, that the values are close to the discussed heat transfer coefficient values shown by Thumm (2010). It was found early during the investigations, that the corner is heating up slightly faster than the middle point, but only the middle thermocouple is taken into account to obtain a more generalized result. 


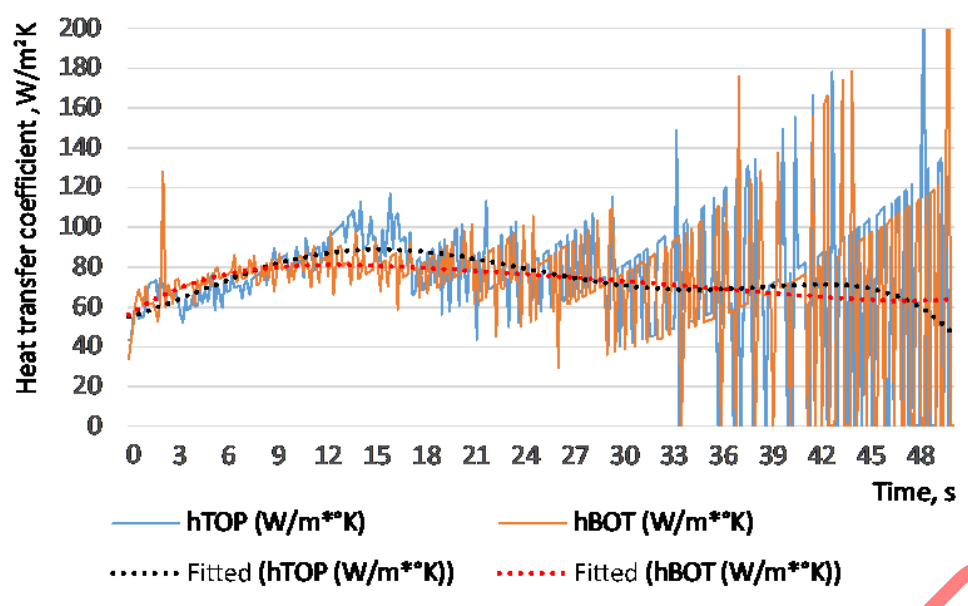

Fig. 6 Obtained heat transfer coefficient values for the top and bottom sides of Box A

The following data presents a comparison between the sides and the box-plate geometries. It is important to note, that the difference between the top and bottom sides are different, but not significantly. The flow regime of the top and bottom sides (Bejan, 1991) (Gerstmann and Griffith, 1978) are assumed to be different, but in practice, this difference may be negligible for presented geometries. It must be noted, that the box sides (which were heated only from one side, practically) resulted in heat transfer coefficient values, which are practically the half, which was recorded on an FR4 board $(\mathrm{h}=171,4)$. The FR4 board was heated from both sides, obviously. The plates of the box were heated from one side and practically no heat flow was present from the other side. The resulted values are in line and range with the literature data on heat transfer coefficients. The results were calculated with the fitted analytic method.

Tab. 1 Obtained average heat transfer coefficient values and the ratio of the sides of Box A

\begin{tabular}{|c|c|c|c|}
\hline Box Sides & Top & Bot & Top/Bot ratio \\
\hline $\boldsymbol{h}, \boldsymbol{W} / \boldsymbol{m}^{2} \boldsymbol{K}$ & 88,9 & 87,2 & $101,80 \%$ \\
\hline
\end{tabular}

During the box immersion, the pressure data was recorded. The additional pressure plot in Figure 6 needs further discussion. As Alaya showed (Alaya, 2019), the particular gage-type sensor works in the lower range of its capabilities. The sensor is not able to hold the signal level in such low hydrostatic pressure condition, however as it is visible in Figure 7, there is a considerable dip of the fitted linear plot at the time point, where the temperature of the box side starts to increase. This dip converts to 2-3 Pa fall, which means that the vapour column collapses $\sim 2 \mathrm{~cm}$. The vapour column recovers in 15-20 sec. The heating seemingly follows the exponential nature described in (3). However, as Figure 5 shows there is an interesting dynamic nature of the heat transfer coefficient - which can be accounted to the consuming of the vapour and needs further investigation in the future. Note, that the plot is extracted from a longer measurement, where the measured box was immersed into the vapour after 2 minutes of initialization. 

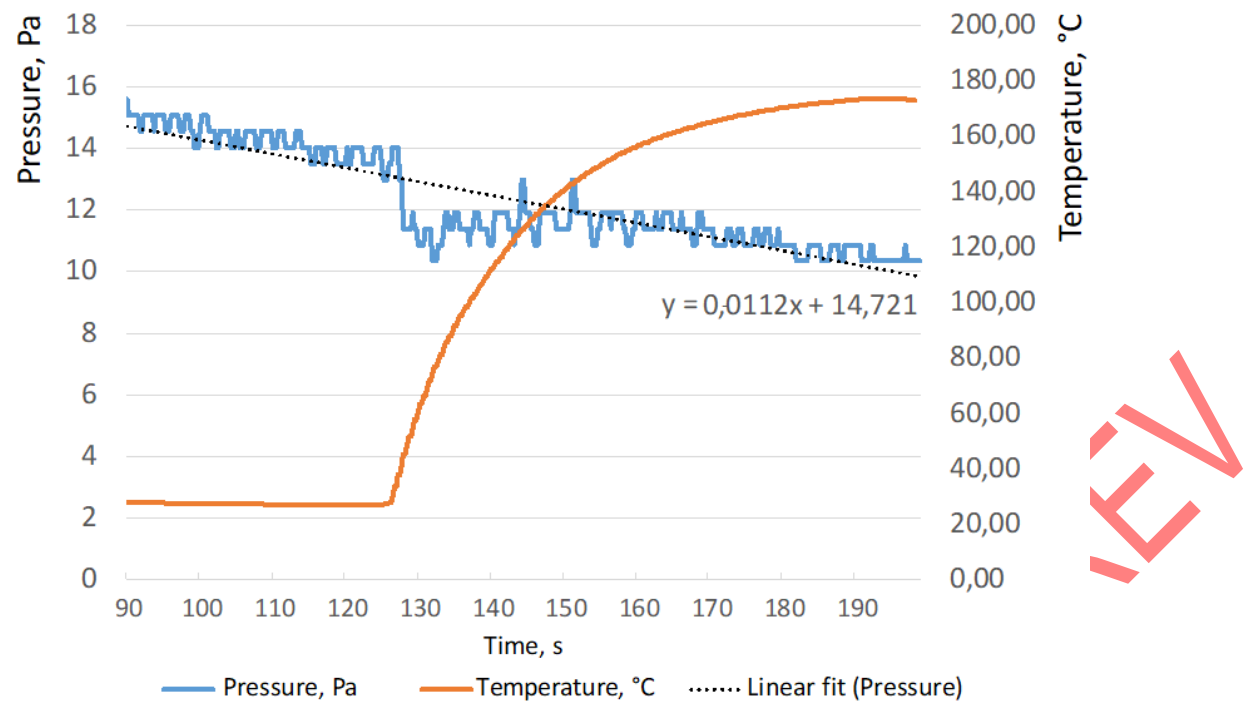

Fig. 7 Hydrostatic pressure drop of the vapour blanket during Box A immersion (Note: when the temperature is rising, the pressure drop is notable from the fitted plot)

\subsection{Heat transfer coefficient investigations with Box B}

From Table 2 it can be stated, that the averaged $h$ is similar to previously presented box heat transfer coefficient values per side. There is a $\sim 8 \%$ difference between vertical and horizontal sides. It is apparent that we can distinguish a difference between the given sides (the vertical sidewalls are heating more intensely - practically due to the faster condensate flow on the wall surfaces). However, we can state, that this difference may be also negligible in selected modelling cases. The results were calculated with the fitted analytic method.

While the box was aimed for separated wall side measurements and calculations, it is must be noted, that the quantitative aspect of the results might not be applied directly on bulk materials, such as SMD components.

As for the pressure measurements, no detectable break was found in the pressure plots during immersion of the sample. This means, that the system was able to hold the saturated levels of vapour inside.

Table 2. Obtained heat transfer coefficients of Box B separated for each side.

\begin{tabular}{|c|c|c|c|c|c|}
\hline Box Sides & TOP & BOT & SIDE1 & SIDE2 & SIDE3 \\
\hline $\boldsymbol{h}, \boldsymbol{W} / \boldsymbol{m}^{\mathbf{2}} \boldsymbol{K}$ & 81,6 & 81,2 & 87,3 & 89,4 & 87,4 \\
\hline
\end{tabular}

Compared to the pressure measurement results in the previous subchapter, it can be concluded, that while there was a significant difference in the sensor's reading, the heat transfer coefficient levels stayed at the same range. This suggests, that while the immersed board consumed a significant volume of vapour around itself and the vapour column collapsed, the saturated vapours around the board recovered fast so that the average heat transfer coefficient stayed similar in case of both box types. It 
also must be noted, that the dynamics of the vapour column and the heat transfer coefficient needs further evaluation - this will be a research path of the future.

\section{Conclusions}

In this paper, the heat transfer was investigated during vapour phase soldering on oriented PCBbased surfaces. While previously the fast explicit board-level modelling was found to be working for bare FR4 PCBs, explicit modelling for three-dimensional bodies, such as small scale SM components, need a revision of the heat transfer coefficients. With a small box model, we separated heat transfer on different sides of a cuboid box body. It was found, that heat transfer on top/bottom is not significantly different in the volume of an experimental VPS oven. It was also found, that heat transfer on side faces are measurably different from the top/bottom, but not significantly. For future reference, it might be a proper method for explicit modelling, to use a uniform $\mathrm{h}$ value for the body, which can describe the heating of the component during VPS soldering, without separating the top, bottom, and side faces of the component. The method and the explicit modelling approach may be used in profile predicting algorithms used in VPS ovens prepared for the challenges and requirements of Industry 4.0 directives for better quality and long term solder reliability.

\section{Acknowledgement}

The research was supported by the National Research, Development and Innovation Office - NKFIH, FK 132186.

\section{References}

Alaya MA; Geczy A; Illes B; Harsányi G; Bušek D (2019) "Advances in pressure sensing for vapour phase soldering process monitoring" Soldering \& Surface Mount Technology, 31:3, pp. 169-175. Bejan A., Film condensation on an upward facing plate with free edges, International Journal of Heat and Mass Transfer 34 (1991) 582-587.

Çengel Y.A., Ghajar, A.J., (2015) "Heat and Mass Transfer", Fifth Ed. McGraw-Hill , New York, USA Dziurdzia B., Sobolewski M., Mikolajek J., (2018) "Convection vs vapour phase reflow in LED and BGA assembly", Soldering \& Surface Mount Technology, Vol. 30 Issue: 2, pp.87-99, https://doi.org/10.1108/SSMT-10-2017-0031

Illés B. (2013) "Modeling Galden layer formation on PCB surface during Vapour Phase Soldering", IEEE 19th International Symposium for Design and Technology in Electronic Packaging (SIITME), Galati, pp. 69-74. doi: 10.1109/SIITME.2013.6743646

Leicht H., Thumm A. (2008) "Today's Vapor Phase SolderingAn Optimized Reflow Technology for Lead Free Soldering", SMTA

Huang L, Zhu L, Wu H, Long X, (2019) "Board-level vapor phase soldering (VPS) with different temperature and vacuum conditions", Multidiscipline Modeling in Materials and Structures, Vol. 15 Issue: 2, pp.353-364, https://doi.org/10.1108/MMMS-04-2018-0082

Livovsky, L. and Pietrikova, A. (2019), "Measurement and regulation of saturated vapour height level in VPS chamber", Soldering \& Surface Mount Technology, Vol. 31 No. 3, pp. 157-162.

https://doi.org/10.1108/SSMT-10-2018-0040

Livovsky L., Pietrikova A., (2017), "Real-time profiling of reflow process in VPS chamber", Solder.

Surf. Mt. Tech, Vol. 29 Iss 1. 
Manncorp, VP260 Vapor Phase Reflow Oven, https://www.manncorp.com/vapor-phase-solderingvp260.html (accessed at: 2019. 10. 01.)

Molhanec M. (2019) "Study of Solder Spreadability at Different Soldering Conditions Using Factorial Experiments", IEEE ISSE Conference 2019, Wroclaw, Poland, DOI: 10.1109/ISSE.2019.8810262

Pavec M., Hirman M., Soukup R. and Hamacek A. (2019) "The Testing of Brass Solderability for Hybrid Sewing Thread", IEEE ISSE Conference 2019, Wroclaw, Poland, DOI:

10.1109/ISSE.2019.8810282

Veselý P., Horynová E., Starý J., Bušek D., Dušek K., Zahradník V., Plaček M., Mach P., Kučírek M., Ježek V., Dosedla M., (2018) "Solder joint quality evaluation based on heating factor", Circuit World, Vol. 44 Issue: 1, pp.37-44, https://doi.org/10.1108/CW-10-2017-0059

Lüngen S., Klemm A., Wohlrabe H., (2015) "Evaluation of the quality of SMDs according to vacuum vapour phase soldering," 2015 38th International Spring Seminar on Electronics Technology (ISSE), Eger, 2015, pp. 218-222. doi: 10.1109/ISSE.2015.7247993

Straubinger D, Bozsóki I, Gál L, Géczy A, (2019) "Scaling of Components for Explicit Modelling of Heat Transfer during Vapour Phase Reflow Soldering", 2019 IEEE 25th International Symposium for Design and Technology in Electronic Packaging (SIITME) - accepted for publication

Synkiewicz B, Skwarek A, Witek K (2015) "Vapour phase soldering used for quality improvement of semiconductor thermogenerators (TEGs) assembly" Mat. Sci. Semicon. Proc., Vol, 38. 346-351.

Thumm A (2010) "Going Lead Free With Vapor Phase Soldering - Lead Free Is Still A Challenge For Major Industries", International Conference on Soldering and Reliability ICSR, SMTA, 2010

Zabel C. (2006) "Condensation Reflow Soldering - The Soldering Process with Solutions for future Technological Demands" ASSCON whitepaper 